\title{
$\Omega$
}

M. Vishnu Sharma

DI

Dept of Respiratory Medicine, AJ Institute of Medical Sciences, Mangaluru, India.

\section{A case of exertional breathlessness}

\section{Case report}

A patient underwent radical neck dissection on right side for cervical lymph node secondary tumours from a primary carcinoma of larynx. He presented with exertional breathlessness 2 weeks after the

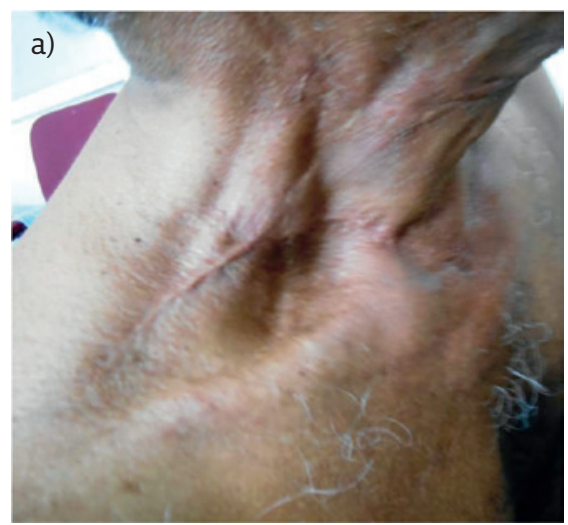

surgery. The results of a physical examination and chest radiography are show in figure 1 . He had no other symptoms.
Cite as: Sharma MV. A case of exertional breathlessness. Breathe 2017; 13: e79-e81.

Figure 1 a) Physical examination. b) Chest radiography.

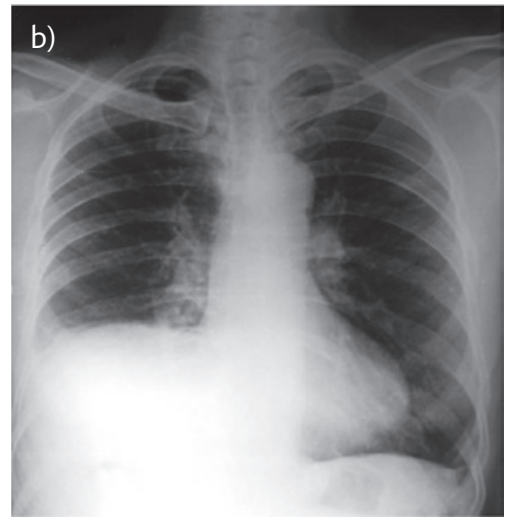

Task 1

What is the most likely diagnosis?
a. Subpulmonic effusion
b. Subdiaphragmatic abscess
c. Right lower lobe collapse
d. Right phrenic nerve palsy
e. Eventration of right dome of diaphragm 


\section{Answer 1}

d. The chest radiograph shows a raised right dome of the diaphragm. All of the possible answers can lead to a raised dome of the diaphragm but with a history of surgery without any other symptoms except exertional breathlessness and with the radiograph showing a smooth contour of right dome, the most likely diagnosis is right phrenic nerve palsy. Unilateral phrenic nerve palsy can lead to exertional breathlessness.
In the neck, the phrenic nerve begins at the lateral border of the anterior scalene muscle. It then continues inferiorly over the anterior surface of the anterior scalene, deep into the prevertebral layer of the deep cervical fascia. Hence, radical neck dissection can lead to phrenic nerve palsy.

\section{Task 2}

1 Which of the following is the best investigation to determine the cause of a raised dome?

a. Computed tomography (CT) of the thorax

b. Lateral decubitus radiography

c. Ultrasound examination of thorax and abdomen

d. Fluoroscopy

e. Magnetic resonance imaging

2 Which of the following statements is wrong?

a. Following cardiac surgery, phrenic nerve paralysis usually occurs on the left side

b. Phrenic nerve paralysis can occur following simple thyroidectomy

c. Phrenic nerve paralysis can lead to hypercapnic respiratory failure

d. Phrenic nerve paralysis can lead to orthopnoea

e. In bronchogenic carcinoma, phrenic nerve paralysis is more common on the left side

3 Which of the following statements is wrong?

a. Diaphragmatic eventration is more common on the left side

b. Morgagni hernia is rare on the left side

c. Trauma can lead to diaphragmatic hernia

d. Noninvasive ventilation is useful in treating hypercapnic respiratory failure due to diaphragmatic dysfunction

e. Spirometry is diagnostic in bilateral diaphragmatic palsy

4 Which of the following is not useful to differentiate eventration of diaphragm from diaphragm paralysis?

a. Phrenic nerve conduction study

b. CT scan thorax and upper abdomen

c. Thoracic ultrasound

d. Fluoroscopy

e. Measurement of trans diaphragmatic pressure 


\section{Answer 2}

1 c. A raised dome can be due to several causes than can be above the diaphragm, in the diaphragm or below the diaphragm. Ultrasound of abdomen and thorax will differentiate all these causes. Besides this, ultrasound is also useful to detect movement of the diaphragm. In phrenic nerve palsy, a "sniff test" may show paradoxical movement of diaphragm.

$2 \mathrm{~b}$. Since the phrenic nerve is deep in the neck, simple thyroidectomy does not lead to phrenic nerve damage. On the left side, the phrenic nerve crosses the aortic arch and vagus nerve, and then descends anteriorly to the left lung root, down the left side of the pericardium. Because of the superficial course of left phrenic nerve, left phrenic nerve palsy is more common than right in bronchogenic carcinoma and following cardiac surgery. Phrenic nerve paralysis is reported in up to $10 \%$ of cases following cardiac surgery. The diaphragm is the chief inspiratory muscle; hence, phrenic nerve paralysis can lead to hypoventilation, hypercapnia and orthopnoea.

3 e. Phrenic nerve paralysis needs to be differentiated from diaphragmatic hernia and eventration. Spirometry is not diagnostic in phrenic nerve palsy. Spirometry will show a restrictive defect, which is nonspecific and can occur in many other conditions. Definitive diagnosis of phrenic nerve palsy is by electromyography (phrenic nerve conduction study).

4 b. ACT scan of the thorax and upper abdomen may be useful to detect the causes for raised dome but can't detect movement and paralysis of diaphragm. Hence CT scan is not useful to differentiate eventration from diaphragm paralysis. In diaphragm paralysis, trans diaphragmatic pressure will be very much reduced. In eventration of diaphragm, trans diaphragmatic pressure will be normal or slightly reduced.
In this patient, ultrasound examination of chest and abdomen confirmed right phrenic nerve palsy. He was a chronic smoker, having stopped smoking 6 months prior. Spirometry showed mild restriction and moderate airway obstruction. The patient had chronic obstructive pulmonary disease due to smoking. He became symptomatic after surgery due to right phrenic nerve palsy. He was treated with inhaled formoterol and tiotropium. He was enrolled for pulmonary rehabilitation. With medications and pulmonary rehabilitation, he became symptomatically better.

\section{Conflict of interest}

None declared. 